\title{
Updated Parameter Estimates for GW190425 Using Astrophysical Arguments and Implications for the Electromagnetic Counterpart
}

\author{
Ryan J. Foley ${ }^{1 \star}$, David A. Coulter ${ }^{1}$, Charles D. Kilpatrick ${ }^{1}$, Anthony L. Piro ${ }^{2}$, \\ Enrico Ramirez-Ruiz ${ }^{1,3}$, Josiah Schwab ${ }^{1}$ \\ ${ }^{1}$ Department of Astronomy and Astrophysics, University of California, Santa Cruz, CA 95064, USA \\ ${ }^{2}$ The Observatories of the Carnegie Institution for Science, 813 Santa Barbara Street, Pasadena, CA 91101, USA \\ ${ }^{3}$ Niels Bohr Institute, University of Copenhagen, Blegdamsvej 17, DK-2100 Copenhagen, Denmark
}

Accepted . Received ; in original form

\begin{abstract}
The progenitor system of the compact binary merger GW190425 had a total mass of $3.4_{-0.1}^{+0.3} \mathrm{M}_{\odot}$ (90th-percentile confidence region, with individual component masses of $m_{1}=2.02_{-0.34}^{+0.58}$ and $\left.m_{2}=1.35_{-0.27}^{+0.26} \mathrm{M}_{\odot}\right)$ as measured from its gravitational wave signal. This mass is significantly different from the Milky Way (MW) population of binary neutron stars (BNSs) that are expected to merge in a Hubble time and from that of the first BNS merger, GW170817. Here we explore the expected electromagnetic signatures of such a system. We make several astrophysically motivated assumptions to further constrain the parameters of GW190425. By simply assuming that both components were NSs, we reduce the possible component masses significantly, finding $m_{1}=1.85_{-0.19}^{+0.27} \mathrm{M}_{\odot}$ and $m_{2}=1.47_{-0.18}^{+0.16} \mathrm{M}_{\odot}$. However if the GW190425 progenitor system was a NS-black hole merger, we find best-fitting parameters $m_{1}=2.19_{-0.17}^{+0.21} \mathrm{M}_{\odot}$ and $m_{2}=1.26_{-0.08}^{+0.10} \mathrm{M}_{\odot}$. For a well-motivated BNS system where the lighter NS has a mass similar to the mass of non-recycled NSs in MW BNS systems, we find $m_{1}=2.03_{-0.14}^{+0.15} \mathrm{M}_{\odot}$ and $m_{2}=1.35 \pm 0.09 \mathrm{M}_{\odot}$, corresponding to only $7 \%$ mass uncertainties and reducing the 90 th-percentile mass range to $32 \%$ and $34 \%$ the size of the original range, respectively. For all scenarios, we expect a prompt collapse of the resulting remnant to a black hole. Examining detailed models with component masses similar to our best-fitting results, we find the electromagnetic counterpart to GW190425 is expected to be significantly redder and fainter than that of GW170817. We find that almost all reported observations used to search for an electromagnetic counterpart for GW190425 were too shallow to detect the expected counterpart. If the LIGO-Virgo Collaboration promptly provides the chirp mass, the astronomical community can adapt their observations to improve the likelihood of detecting a counterpart for similarly "high-mass" BNS systems.
\end{abstract}

Key words: gravitational waves, stars: black holes, stars: neutron

\section{INTRODUCTION}

The detection of gravitational waves (GWs) from the merger of compact objects has unveiled new populations of black holes (BHs; Abbott et al. 2016; The LIGO Scientific Collaboration et al. 2018) and now neutron stars (NSs). Observations of GW190425, the second probable BNS merger detected, by the LIGO-Virgo Collaboration (LVC; The LIGO Scientific Collaboration \& the Virgo Collaboration 2020,

* E-mail:foley@ucsc.edu hereafter LVC20) indicate that it was likely a binary NS (BNS) merger, but with the total mass of the system, $3.4_{-0.1}^{+0.3} \mathrm{M}_{\odot}$, being significantly larger than any of the known BNS systems in the Milky Way (MW; e.g., Özel \& Freire 2016; Farrow et al. 2019). This puzzling system has already led to questions about its formation scenario (Romero-Shaw et al. 2020; Safarzadeh et al. 2020).

GW190425 is especially intriguing since the first BNS merger, GW170817, had a total mass consistent with the MW BNS population (Abbott et al. 2017a). The relative rates of GW170817-like and GW190425-like events are com- 
parable (LVC20), indicating that significant selection effects, dramatically different delay times, and/or significant environmental differences are necessary to produce such mergers at similar rates yet have no "high-mass" systems in the MW sample.

Ideally, we would have detected an electromagnetic (EM) counterpart to GW190425 to pinpoint its location and glean additional information about the system. For GW170817, Coulter et al. (2017) discovered the optical counterpart, AT 2017gfo (also known as Swope Supernova Survey 2017a or SSS17a), giving way to numerous studies in the areas of high-energy astrophysics (Goldstein et al. 2017; Murguia-Berthier et al. 2017), nuclear physics (Margalit \& Metzger 2017; Abbott et al. 2018; Annala et al. 2018; Radice et al. 2018), general relativity (Barack et al. 2019), $r$ process nucleosynthesis (Kasen et al. 2017; Kilpatrick et al. 2017; Rosswog et al. 2018), compact-object formation channels (Blanchard et al. 2017; Levan et al. 2017; Pan et al. 2017; Ramirez-Ruiz et al. 2019), and cosmology (Abbott et al. 2017b; Guidorzi et al. 2017).

However, no such counterpart has been confidently discovered for GW190425 (Antier et al. 2019; Coughlin et al. 2019; Lundquist et al. 2019; Hosseinzadeh et al. 2019), perhaps partly because of the large localization area for GW190425 (90th-percentile region of 8,284 $\mathrm{deg}^{2}$; LVC20), high distance $\left(159_{-71}^{+69} \mathrm{Mpc}\right)$, and that a significant fraction of the localization region was either Sun constrained or at low Galactic latitudes. An alternative possibility is that the counterpart was intrinsically faint, as indicated by the luminosity function of kilonovae associated with short gammaray bursts (Gompertz et al. 2018; Ascenzi et al. 2019).

LVC20 performed a sophisticated and detailed analysis of the GW strain data and provided posterior distributions for parameters that describe the system ${ }^{1}$. Their approach was to make minimal assumptions and allow the data constrain the parameter estimates. However, part of the resulting allowed parameter space is inconsistent with separate astrophysical constraints on NSs and BHs. Here, we apply additional astrophysical constraints to significantly reduce the allowed parameter space for GW190425. With the more constraining data, we examine the characteristics of a possible EM counterpart, finding that it likely was significantly fainter and redder than AT 2017gfo.

We introduce our astrophysically motivated assumptions and apply them to the GW190425 data in Section 2. In Section 3, we explore how the updated parameter estimates affect the properties of an EM counterpart. In Section 4, we discuss additional implications of the GW190425 system. We conclude in Section 5.

\section{PHYSICALLY MOTIVATED SYSTEMS}

In this section, we make several arguments to reduce the parameter space of possible progenitor systems for GW190425. We first separate different scenarios into BNS and NSBH mergers.

In the former scenario, high spins and a particularly high mass for $m_{1}$ are ruled out by physical arguments. We

\footnotetext{
${ }^{1}$ https://dcc.ligo.org/LIGO-P2000026/public
}

are further able to restrict the parameter space by examining two possible progenitor systems, an equal-mass system and a system including a NS whose mass is consistent with the non-recycled NSs in MW BNS systems (a mass close to a Chandrasekhar mass).

Similar physical arguments require that the NSBH scenario have an extreme mass ratio. Again, assuming that the lighter object is consistent with the MW BNS population provides stringent constraints.

\subsection{Is GW190425 a BNS or NSBH Merger?}

Given the total mass of the system, GW190425 is likely a BNS merger, but an NSBH merger cannot be ruled out by the GW data alone (see also Han et al. 2020). Unless there is a population of very low-mass BHs (i.e., $M<1.7 \mathrm{M}_{\odot}$ ), GW190425 was not a BBH merger, and we do not further consider that scenario.

We distinguish if the primary object is a $\mathrm{BH}$ based on its mass. We assume that if $m_{1} \leqslant M_{\mathrm{TOV}}$, the TolmanOppenheimer-Volkoff (TOV) mass (Oppenheimer \& Volkoff 1939; Tolman 1939), then the primary is a NS. We assume it is a BH if $m_{1}>M_{\mathrm{TOV}}$. Because we do not precisely know the equation of state for nuclear matter, we must constrain this maximum mass through observations. We use two pulsar mass measurements for this constraint. Of well-measured pulsars, the most important for this quantity is the millisecond pulsar J0740+6620, which has the highest measured mass, $M_{0740}=2.14_{-0.09}^{+0.10} \mathrm{M}_{\odot}$ (Cromartie et al. 2020). We also include J0348+0432 with a mass of $M_{0348}=2.01 \pm 0.04 \mathrm{M}_{\odot}$, which because of its small mass uncertainty is particularly important for excluding the low-mass tail. Critically for the NSBH case, we assume $M_{\text {TOV }} \geqslant M_{0704}$ and $M_{0348}$.

We also assume $M_{\mathrm{TOV}}$ is less than the maximum value determined from requiring a hypermassive NS after the merger of GW170817. Several studies have performed similar analyses (Margalit \& Metzger 2017; Shibata et al. 2017, 2019; Ruiz et al. 2018; Ai et al. 2019), all finding $M_{\text {TOV }} \lesssim 2.1$ - $2.3 M_{\odot}$ (at 90th-percentile confidence). We use the distribution of limits from these sources to produce a onesided Gaussian distribution to predict the upper limit for $M_{\text {TOV }}$, finding it is best fit with a central value of 2.10 and a width of $0.06 \mathrm{M}_{\odot}$, corresponding to a 90 th-percentile limit of $2.23 \mathrm{M}_{\odot}$. Notably this distribution is also consistent with the measured mass of the pulsar J0740+6620.

Additionally, we assume that the less massive component of the GW190425 system is a NS, requiring $m_{2} \geqslant$ $M_{\text {min, NS }}$, the minimum mass of a NS. For this minimum mass, we adopt the mass of the unseen companion in J0453+1559, $M=1.174 \mathrm{M}_{\odot}$ (Martinez et al. 2015). Suwa et al. (2018) suggest a stellar evolution pathway for producing such a low-mass NS, although see Tauris \& Janka (2019) for an alternative scenario in which this object is instead a white dwarf.

Starting with the PhenomPv2NRT (Dietrich et al. 2019) high-spin posterior distributions (LVC20, corresponding to their preferred model), we resample the posterior distributions with additional conditions. Whenever we assume a component is a NS, we require that its mass be $M_{\text {min, NS }} \leqslant M \leqslant M_{\text {TOV }}$ max. When we assume a component is a black hole, we assume $M>M_{\mathrm{TOV}}$, min 


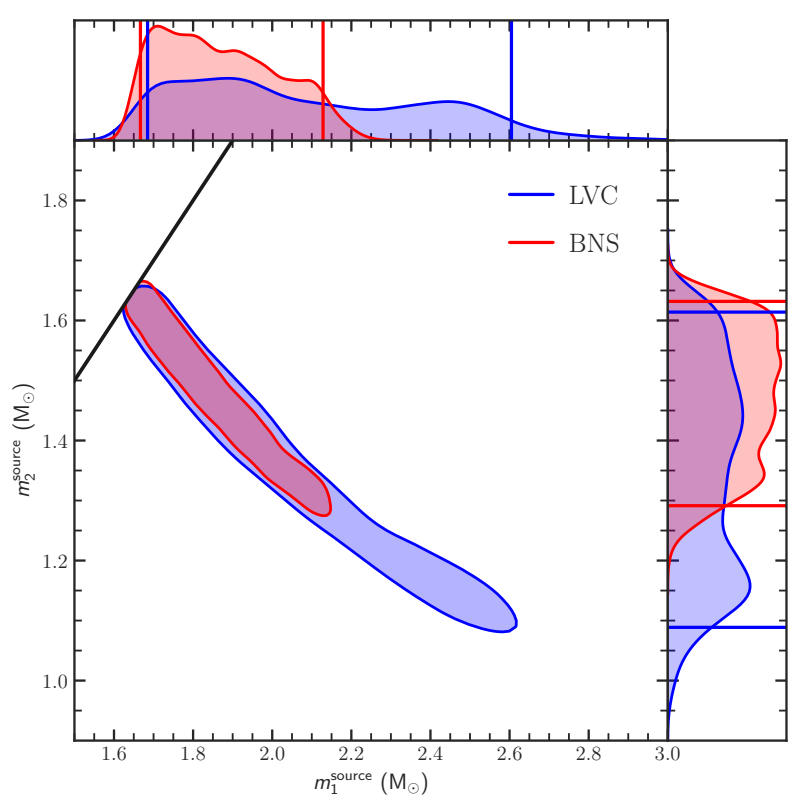

Figure 1. Posterior distributions of the component masses $m_{1}$ and $m_{2}$ in the source frame. The blue contours represent the LVC20 high-spin prior distributions, while the red contours are recovered by assuming GW190425 was a BNS merger. The solid black line represents systems with equal mass. Vertical lines in the one-dimensional plots enclose $90 \%$ of the probability with the distributions scaled to have the same total area.

We additionally apply a prior on the spin for any assumed NS component. Gravitational wave data does not directly constrain the spin, but rather

$$
\chi=\frac{c S}{G m^{2}},
$$

where $S$ is the amplitude of the spin vector and $m$ is the NS mass. This results in a degeneracy between NS spin and mass. Equivalently, one can write $\chi$ as

$$
\chi=\frac{I \Omega c}{G m^{2}},
$$

where $I$ is the moment of inertia and $\Omega$ is the spin frequency. For a NS with a millisecond period and reasonable NS equations of state, we find a maximum $\chi$ of $\sim 0.2$, significantly less than the "high-spin" prior of $\chi<0.89$ used by LVC20. To be conservative, we apply a prior of $\chi<0.4$ for any NS component.

Without any assumptions about the nature of the progenitor system, LVC20 found a total system mass of $3.39_{-0.11}^{+0.32} \mathrm{M}_{\odot}$ and component masses of $m_{1}=2.02_{-0.34}^{+0.58} \mathrm{M}_{\odot}$ and $m_{2}=1.35_{-0.27}^{+0.26} \mathrm{M}_{\odot}$ (all 90th-percentile confidence regions and measured in the source frame). If we assume that GW190425 was a BNS system, the allowed parameter space reduces to $M_{\text {tot }}=3.33_{-0.06}^{+0.10} \mathrm{M}_{\odot}$ and component masses of $m_{1}=1.85_{-0.19}^{+0.27} \mathrm{M}_{\odot}$ and $m_{2}=1.47_{-0.18}^{+0.16} \mathrm{M}_{\odot}$, making the total mass uncertainty only $37 \%$ that of its original measurement. We present the updated mass constraints in Figure 1 and Table 1.

As noted by $\mathrm{LVC} 20, m_{1}$ and $\chi$ are highly correlated for GW190425. Not including the $\chi$ constraint results in a $\chi$ distribution for the more massive component that contains very little probability at $>0.4$. However, $\sim 5 \%$ of the $\chi$

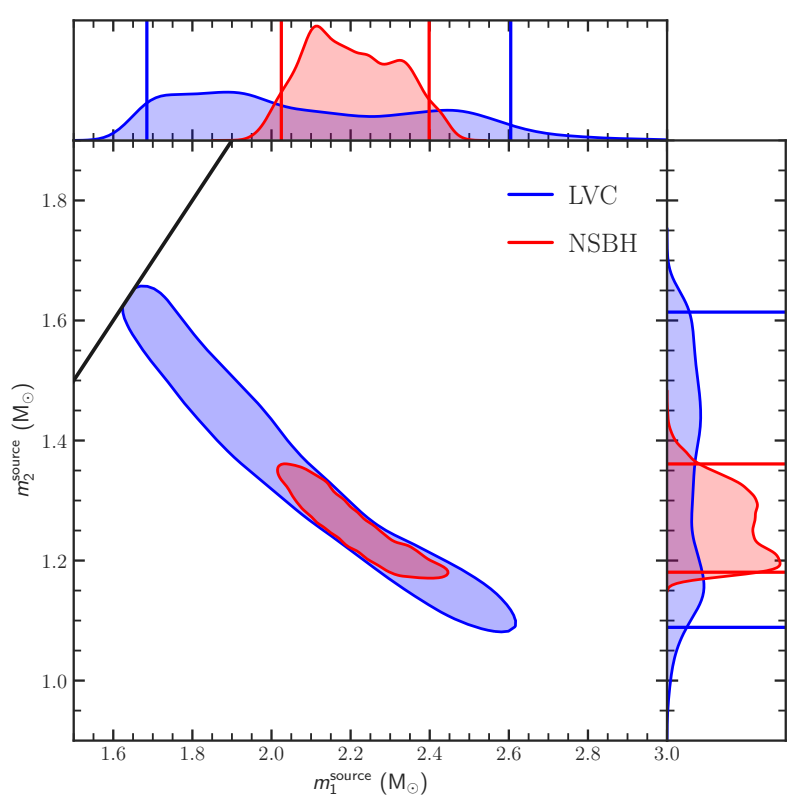

Figure 2. Same as Figure 1, but where the solid red contours are recovered by assuming GW190425 was an NSBH merger.

distribution for the less-massive component is at $>0.4$. We therefore include this condition despite its limited predictive power.

For the NSBH system, we can apply a separate constraint that there is no tidal deformation for the more massive component (i.e., $\Lambda_{1}=0$ ). We performed the analysis both with and without this constraint, finding no significant difference. We therefore do not include the constraint.

If we assume that GW190425 was a NSBH merger, we find $M_{\text {tot }}=3.46_{-0.09}^{+0.13} \mathrm{M}_{\odot}$ and component masses of $m_{1}=$ $2.19_{-0.17}^{+0.21} \mathrm{M}_{\odot}$ and $m_{2}=1.26_{-0.08}^{+0.10} \mathrm{M}_{\odot}$, reducing the total mass uncertainty to $56 \%$ that of its original uncertainty. We present the updated mass constraints in Figure 2.

\subsection{A Chandrasekhar-mass Neutron Star}

The individual NSs in Milky Way BNS systems have a mass distribution centered at $1.33 \mathrm{M}_{\odot}$ and a standard deviation of $0.09 \mathrm{M}_{\odot}\left(\ddot{O ̈}_{z e l} \&\right.$ Freire 2016), indicating that such NSs have a preference for masses similar to the Chandrasekhar mass $^{2}$. Separately, core-collapse modeling indicates that many remnants should be close to a Chandrasekhar mass (Sukhbold et al. 2016). This is especially expected in the case of an electron-capture supernova (Kitaura et al. 2006) or the accretion-induced collapse of a WD (Timmes et al. 1996). There is a correlation between NS mass and kick velocity (Podsiadlowski et al. 2004), providing an additional reason why at least the second NS in a merging BNS system would have a mass on the low-end of viable NS masses.

Given the total mass of the GW190425 system, having

2 We remind the reader that while the Chandrasekhar mass might be related to the birth of a NS, it does not physically constrain its mass. However, we use the name as a reference to the approximate mass of the population of MW BNSs. 


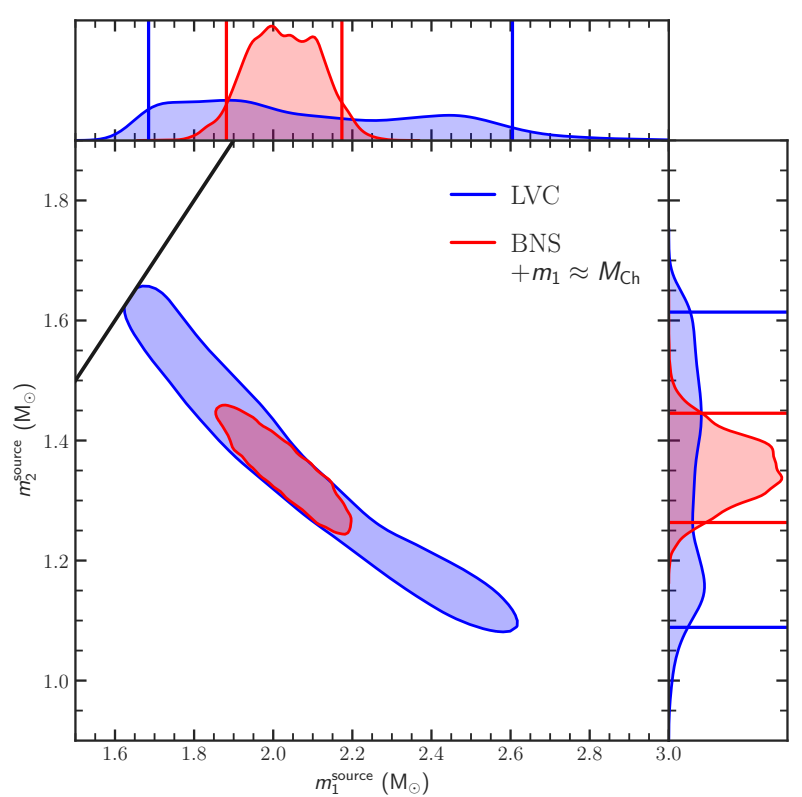

Figure 3. Same as Figure 1, but where the solid red contours are recovered by assuming GW190425 was a BNS merger where the lighter object was NS drawn from the Farrow et al. (2019) non-recycled NS mass distribution.

one Chandrasekhar-mass NS would imply that the second NS would have $m \approx 2 \mathrm{M}_{\odot}$. Such a massive NS is obviously not observed in Milky Way BNS systems, however, NSs that massive are known, including in NS-WD systems (Antoniadis et al. 2016).

Combined, a $1.4+2.0 \mathrm{M} \odot$ system is a natural choice for the GW190425 progenitor system. Such masses are consistent with either a BNS or NSBH system and is close to the best-fitting masses from GW data alone $\left(1.35+2.02 \mathrm{M}_{\odot}\right)$. We note, however, that such a system is somewhat in tension with the low-spin prior $(\chi<0.05)$ assumed by LVC20.

Motivated by these considerations, we use the bestfitting population to the non-recycled pulsars in MW BNS systems as determined by Farrow et al. (2019). They find that a uniform prior with a lower bound of $1.16_{-0.02}^{+0.01} \mathrm{M}_{\odot}$ and an upper bound of $1.42_{-0.02}^{+0.04} \mathrm{M}_{\odot}$ is a better fit than a Gaussian distribution for the non-recycled NSs. Using this prior and assuming a BNS system, we find $m_{1}=2.03_{-0.14}^{+0.15} \mathrm{M}_{\odot}$ and $m_{2}=1.35 \pm 0.09 \mathrm{M}_{\odot}$. While these values are consistent with the LVC20 values and from assuming a BNS system, the mass ranges are significantly reduced. We present the updated mass constraints in Figure 3.

In this scenario, we find a mass ratio of $q=0.67_{-0.08}^{+0.10}$, indicating a system far from equal mass, but intriguingly close to the best-fitting value without any astrophysical assumptions $\left(q=0.67_{-0.25}^{+0.29}\right.$. Additionally, we find that the luminosity distance is somewhat, but insignificantly, larger with these assumption $\left(D_{L}=171_{-76}^{+66} \mathrm{Mpc}\right.$ with and $D_{L}=$ $159_{-71}^{+69}$ Mpc without).

For the NSBH scenario, the posterior distributions do not change significantly when including this additional assumption. As can be seen from Figure 2, the secondary mass is already constrained to within the Farrow et al. (2019) mass distribution with the basic NSBH assumptions.

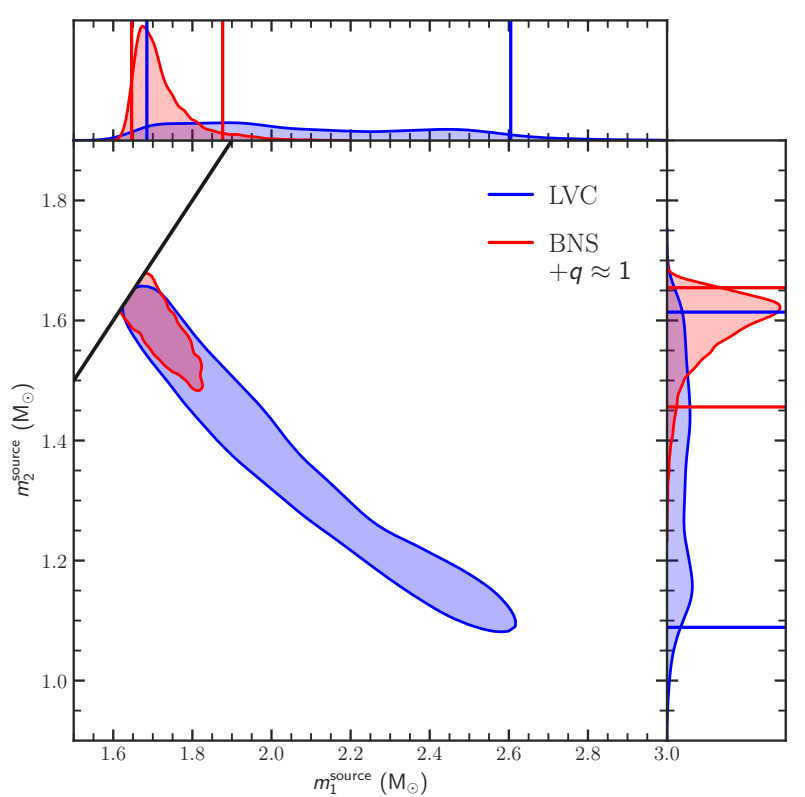

Figure 4. Same as Figure 1, but where the solid red contours are recovered by assuming GW190425 was a near-equal-mass BNS merger.

\subsection{An Equal-mass System}

All known MW BNS systems have mass ratios of $q \approx 1$, with the lowest measured value for a system expected to merge in a Hubble time of $0.75 \pm 0.05$ (Ferdman \& PALFA Collaboration 2018) and the second-lowest being $0.92 \pm 0.01$ (Ferdman et al. 2014). While none have a total mass of $>2.9 \mathrm{M}_{\odot}$, there is a clear preference for equal-mass systems in this population. Additionally, the GW170817 BNS system and nearly all $\mathrm{BBH}$ systems are also consistent with their components having equal mass (Abbott et al. 2019; The LIGO Scientific Collaboration et al. 2018). It is clear that Nature produces many compact binary systems with near-equal mass.

We use the distribution of mass ratios from the MW BNS systems as determined by Farrow et al. (2019) to investigate the implications for GW190425. The 90th-percentile prior lower bound on $q$ is 0.836 . This configuration requires a low spin to be consistent with the GW data.

Under the assumption that GW190425 was as BNS with roughly equal-mass components, the parameter space is especially constrained. We find the components had masses of $m_{1}=1.70_{-0.06}^{+0.17} \mathrm{M}_{\odot}$ and $m_{2}=1.60_{-0.14}^{+0.05} \mathrm{M}_{\odot}$. We present the updated mass constraints in Figure 4 . The mass ratio is constrained to $q=0.94_{-0.16}^{+0.05}$ and $\chi_{\mathrm{eff}}=0.02_{-0.02}^{+0.03}$, consistent with expectations and the low-spin prior from LVC20.

\section{ASTROPHYSICAL IMPLICATIONS}

In this section, we detail the implications of the tighter priors assumed in Section 2.

\subsection{Remnant}

The source-frame chirp mass for GW190425 of $\mathcal{M}=$ $1.437_{-0.020}^{+0.022} \mathrm{M}_{\odot}$ is sufficiently large that if GW190425 came 
from a BNS system, it likely resulted in a prompt collapse to a black hole (see e.g., Piro et al. 2017). We can assess this by examining the remnant mass compared to the TOV mass and the radius of a $1.6 \mathrm{M}_{\odot} \mathrm{NS}, R_{1.6}$. Following Bauswein et al. (2013) and Bauswein et al. (2017), we expect prompt collapse if

$$
M_{\mathrm{tot}}>M_{\mathrm{thresh}} \approx\left(2.38-3.606 \frac{G M_{\mathrm{TOV}}}{c^{2} R_{1.6}}\right) M_{\mathrm{TOV}} .
$$

The remnant should have underwent prompt collapse if $M_{\text {tot }}$ is larger than the maximum value of $M_{\text {thresh }}$ given current constraints on $M_{\mathrm{TOV}}$ and $R_{1.6}$. For $M_{\mathrm{TOV}}=2.17 \mathrm{M}_{\odot}($ Margalit \& Metzger 2017) and $R_{1.6}=13.5 \mathrm{~km}$ (De et al. 2018), we find $M_{\text {thresh, max }}=3.3 \mathrm{M}_{\odot}$.

The remnant for GW190425 likely had a mass similar to the threshold mass. From GW data alone, GW190425 had $M_{\text {tot }}=3.39_{-0.11}^{+0.32} \mathrm{M}_{\odot}$. However, we should examine only BNS mergers since NSBH systems will never have a NS remnant, even if short lived. In Section 2.1, we found that if GW190425 is a BNS system, it had $M_{\text {tot }}=3.33_{-0.06}^{+0.10} \mathrm{M}_{\odot}$. Assuming that the lighter component had a mass similar to the Galactic mass distribution, we find GW190425 had $M_{\text {tot }}=3.38_{-0.07}^{+0.08} \mathrm{M}_{\odot}$. As noted below, it is unlikely that it ejected more than $0.04 \mathrm{M}_{\odot}$. Additionally, $M_{\text {thresh }}$ may be as low as $\sim 2.7 \mathrm{M}_{\odot}$ given current constraints on $M_{\mathrm{TOV}}$ and $R_{1.6}$. We therefore believe it is likely that the remnant of GW190425 promptly collapsed to a black hole.

This basic analysis is consistent with that of LVC20, which found a $97 \%$ chance of a prompt collapse using recent simulations, a range of equations of state, and their highspin priors. A prompt collapse implies that GW190425 was likely accompanied by a short gamma-ray burst (MurguiaBerthier et al. 2014), which was not confidently seen (although, see Pozanenko et al. 2019); however, the data are not constraining (Song et al. 2019).

For the remainder of this work, we assume that if GW190425 was a BNS merger, it underwent a prompt collapse. Even if it produced a hypermassive NS, it would have been sufficiently massive to collapse quickly, making the resulting ejecta qualitatively similar to the prompt-collapse scenario.

\section{$3.2 \quad$ Ejecta}

Rosswog (2013) produced several dynamical merger models of compact binary mergers (see also Korobkin et al. 2012). The models include both BNS and NSBH mergers over a large mass range. We use these models to estimate the amount of dynamical ejecta and its velocity. In particular, the $1.4+2.0$ and $1.6+1.6$ BNS merger models produced 0.039 and $0.020 \mathrm{M}_{\odot}$ of ejecta with an average velocity of 0.15 and $0.11 c$, respectively. There are no low-mass NSBH models, but the $1.4+5.0 \mathrm{NSBH}$ (corresponding to the leastmassive NSBH merger presented) model produced $0.024 \mathrm{M}_{\odot}$ with an average velocity of $0.15 c$, and lower-mass models are expected to produce less ejecta with a smaller average velocity.

Other studies have examined a range of NS masses (and equations of state), but do not fully cover the range of possible systems for GW190425 (e.g., Bauswein et al. 2013; Radice et al. 2017), and we therefore focus on the Rosswog (2013) results for consistency. We note, however, that these alternative simulations generally find ejecta masses that are smaller by a factor of $\gtrsim 2$ depending primarily on the assumed NS equation of state. Because of these differences, we consider the Rosswog (2013) models as having optimistic ejecta masses and GW190425 may have ejected significantly less material than corresponding models.

We argue in Section 3.1 that GW190425 likely underwent a prompt collapse to a $\mathrm{BH}$. In this scenario, all ejecta (regardless of exact origin) is expected to have low electron fraction, $Y_{e}$.

\subsection{Electromagnetic Counterparts}

AT 2017gfo, the optical counterpart to GW170817 (Coulter et al. 2017), was relatively bright at all optical wavelengths, peaking at $M \approx-16 \mathrm{mag}$ (Andreoni et al. 2017; Cowperthwaite et al. 2017; Drout et al. 2017; Evans et al. 2017; Kasliwal et al. 2017; McCully et al. 2017; Smartt et al. 2017; Tanvir et al. 2017; Utsumi et al. 2017; Valenti et al. 2017). It faded extremely quickly in blue bands but was longer lived in the NIR (e.g., Siebert et al. 2017). This broad-band behavior requires ejecta with a distribution of lanthanide fractions, $X_{\text {lan }}$ that is often parameterized as separate components each with a single lanthanide fraction (e.g., Drout et al. 2017; Villar et al. 2017).

The lanthanide fraction is directly related to the electron fraction, $Y_{e}$, of the material, with $Y_{e} \lesssim 0.25$ material producing a significant fraction of lanthanides (Lippuner \& Roberts 2015). Ejecta created through separate processes during the merger are expected to have different $Y_{e}$ (Metzger \& Fernández 2014) with the tidal debris having $Y_{e} \lesssim 0.1$ (e.g., Rosswog 2005) and a neutrino-irradiated disk wind having $Y_{e} \gtrsim 0.25$ (Fernández \& Metzger 2013). The amount of ejecta from the disk wind and its $Y_{e}$ depends critically on the mass of the remnant and lifetime of a possible hypermassive or supramassive NS (Metzger \& Fernández 2014). The bright blue colors of AT 2017 gfo require a short-lived hypermassive NS (Margalit \& Metzger 2017; Murguia-Berthier et al. 2017).

As discussed in Section 3.1, GW190425 likely underwent a prompt collapse and had low $Y_{e}$, and thus the ejecta should have a high $X_{\text {lan }}$. All models considered exclusively produce low $-Y_{e}$ ejecta that correspond to high lanthanide fractions of $\log X_{\operatorname{lan}} \approx-2$.

Kasen et al. (2017) produced light curves for kilonovae with a range of $M_{\mathrm{ej}}, v$, and $X_{\mathrm{lan}}$. Kilpatrick et al. (2017) used a combination of two of these models corresponding to lowlanthanide and high-lanthanide components to match the light curves of AT 2017gfo. This is confirmation that kilonova observations can be reproduced by theoretical models.

For comparison, we examine light curves for a kilonova with $M_{\mathrm{ej}}=0.025,0.03$, and $0.04 \mathrm{M}_{\odot} ; v=0.1,0.15$, and $0.15 c$; and $\log X_{\text {lan }}=-2$ (for all models), respectively. These models are similar to the Rosswog (2013) models for $1.6+1.6,1.2+2.0$, and $1.4+2.0 \mathrm{M}_{\odot}$ BNS mergers. The first two models are also somewhat similar to the $1.4+5.0 \mathrm{M}_{\odot}$ NSBH merger. We display the light curves corresponding to the $1.4+2.0 \mathrm{M}_{\odot}$ the model and the Kilpatrick et al. (2017) model for AT 2017gfo in Figure 5. While the NIR light curves of these two models are nearly identical, the optical light curves are significantly different with the $1.4+2.0 \mathrm{M}_{\odot}$ BNS merger producing a kilonova with significantly less op- 


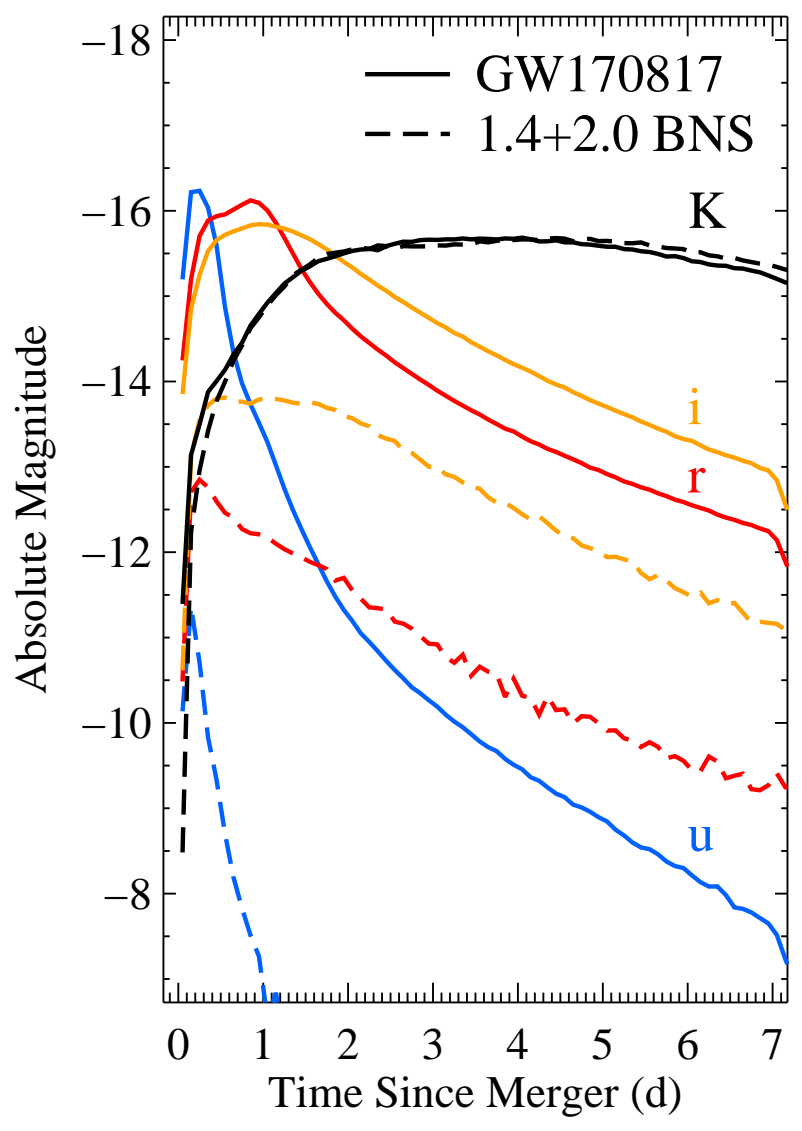

Figure 5. uriK (blue, red, orange, and black curves) absolute light curves for kilonova models (Kasen et al. 2017). The solid curve is a model matched to the observations of GW170817 (Kilpatrick et al. 2017) and contains both blue, low lanthanide fraction and red, high lanthanide fraction components. The dashed curves represent a model with $M_{\mathrm{ej}}=0.04 \mathrm{M}_{\odot}, v=0.15 c$, and $\log X_{\text {lan }}=-2$, similar parameters to hydrodynamical models of a $1.4+2.0 \mathrm{M}_{\odot}$ BNS merger (Rosswog 2013), a likely scenario for GW190425.

tical luminosity. The other models, which have less ejecta mass, produce significantly less luminous kilonovae, with the $1.6+1.6 \mathrm{M}_{\odot}$ model being $\sim 1$ mag fainter in $i$ at peak than the $1.4+2.0 \mathrm{M}_{\odot}$ model. The $1.4+2.0 \mathrm{M}_{\odot}$ model is the most luminous of models consistent with GW190425.

In Figure 6, we display the differences between the two models. As seen in Figure 5, the $K$-band light curves are nearly identical. However, the $1.4+2.0 \mathrm{M}_{\odot}$ BNS merger kilonova is $\sim 6,3$, and 2 mag less luminous than GW170817 in uri over the first week after the merger.

\section{DISCUSSION}

\subsection{Parameters}

The astrophysically motivated assumptions reduced the uncertainties on several parameters. As these assumptions were never in tension with the observations, the parameters did not shift dramatically from the LVC20 results.

While our assumptions significantly affected the massrelated parameters (each component mass, total mass, and

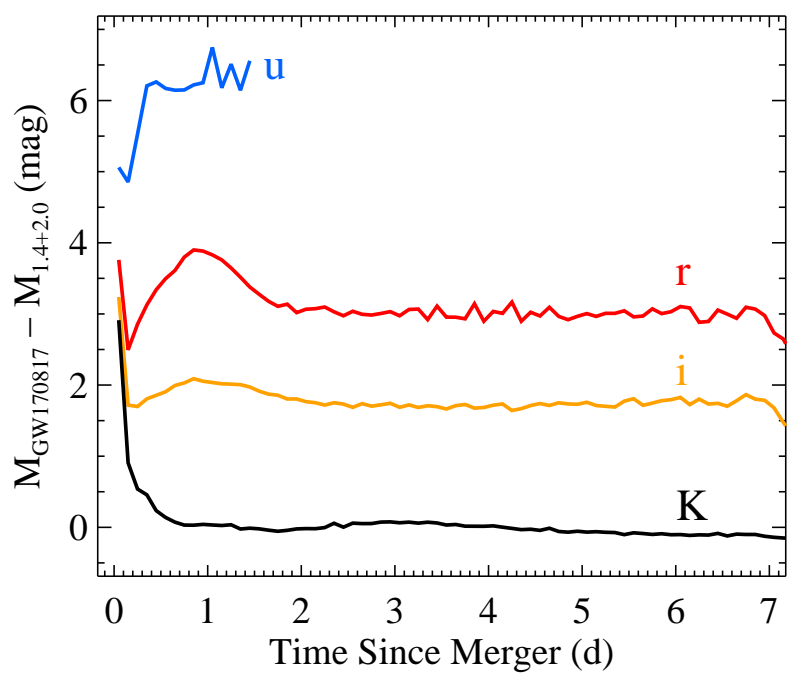

Figure 6. Difference in absolute magnitude between kilonova models (Kasen et al. 2017) for GW170817 (Kilpatrick et al. 2017) and a model with $M_{\mathrm{ej}}=0.04 \mathrm{M}_{\odot}, v=0.15 c$, and $\log X_{\operatorname{lan}}=-2$, similar parameters to hydrodynamical models of a $1.4+2.0 \mathrm{M}_{\odot}$ BNS merger (Rosswog 2013), a likely scenario for GW190425. The blue, red, orange, and black curves correspond to the uriK bands, respectively. The $u$ band is shown only for the first $1.5 \mathrm{~d}$ after merger, after which time it is so faint in the $1.4+2.0 \mathrm{M}_{\odot}$ model that there are insufficient photon statistics to create a reasonable light curve. In the optical bands, we expect GW190425 to be several magnitudes less luminous than GW170817.

mass ratio), moderately affected $\chi_{\text {eff }}$, and had a minor effect on the distance, it did not change most parameters such as the tidal deformation or inclination. As such, our study cannot improve our understanding of the nuclear equation of state or more precisely constrain its location.

Nevertheless, the mass and spin parameters are important for understanding the origin of the system and its ultimate fate. The BNS, BNS with $m_{2} \approx M_{\mathrm{Ch}}$, and NSBH scenarios are all consistent with $m_{1}=2.0 \mathrm{M}_{\odot}, m_{2}=1.3 \mathrm{M}_{\odot}$, and $\chi_{\text {eff }}=0.05$. These values are consistent with a separate analysis specifically examining the NSBH case (Han et al. 2020), although the values presented here are more precise. The $q \approx 1$ scenario disfavors such a system and instead prefers $m_{1} \approx m_{2} \approx 1.65 \mathrm{M}_{\odot}$ and $\chi_{\mathrm{eff}}=0.02$.

For all scenarios except the equal-mass case, we find a relatively high $\chi_{\text {eff }}$ that is inconsistent with MW BNS systems. Such a constrained system (masses known to $\sim 10 \%$ and spin constraints) should guide explorations of possible pathways to creating such a system. Similarly, there must only be a few possible ways to create a low-spin, high-butequal-mass system.

\subsection{Searching for an EM Counterpart to GW190425}

In the minutes after the merger of GW190425, the LVC publicly announced its detection as a likely BNS merger ${ }^{3}$ with a

3 This is not an assessment of the structure of the individual components. The classification scheme used considers any system where each component has $m<3 \mathrm{M}_{\odot}$ a BNS. 
low false-alarm rate (1 per 69,834 years; Ligo Scientific Collaboration \& VIRGO Collaboration 2019a). They quickly released a three-dimensional probability map of the location of the GW emission with a two-dimensional 90th-percentile localization region of $10,183 \mathrm{deg}^{2}$ which was revised about a day later to having a size of $7,461 \mathrm{deg}^{2}$ (Ligo Scientific Collaboration \& VIRGO Collaboration 2019b); the final map released in 2020 January has a $8,284 \mathrm{deg}^{2}$ two-dimensional 90th-percentile localization region (LVC20).

Although this region covers $\sim 1 / 5$ of the entire sky, several groups searched for an EM counterpart (e.g., Antier et al. 2019; Coughlin et al. 2019; Hosseinzadeh et al. 2019; Lundquist et al. 2019). Collectively, $\sim 50 \%$ of the twodimensional localization area was observed in the days following the merger.

We highlight three sets of observations: Coughlin et al. (2019) observed $8000 \mathrm{deg}^{2}$ in $\mathrm{gr}$ with a limiting magnitude of $\sim 21$ and $2400 \mathrm{deg}^{2}$ in $J$ with a limiting magnitude of $\sim 15.5$; Hosseinzadeh et al. (2019) observed 67 galaxies in $i$ with a limiting magnitude of $\sim 22.5$; and Tohuvavohu et al. (2019) observed 408 galaxies in $u$ with a limiting magnitude of 21.1 . These observations correspond to the widest/reddest, deepest, and bluest searches, respectively.

GW190425 has a distance of $159_{-71}^{+69} \mathrm{Mpc}$, corresponding to a distance modulus of $36.01_{-1.28}^{+0.78}$ mag. Assuming no dust extinction (in the MW, host galaxy, or circumstellar environment), the observations listed above have absolute magnitude limits of about $-15.0 \mathrm{mag}$ in wide-field $\mathrm{gr},-20.5 \mathrm{mag}$ in wide-field $J,-13.5 \mathrm{mag}$ in the deepest $i$, and $-14.9 \mathrm{mag}$ in $u$, with an uncertainty of $\sim 1$ mag depending on the distance to GW190425. Taking the distance into account, we find that the Coughlin et al. (2019) $g_{-}, r_{-}$, and $J$-band observations could detect a GW190425-like 1.4+2.0 $\mathrm{M}_{\odot}$ BNS merger to $D=46,59$, and $15 \mathrm{Mpc}$, respectively, indicating that those observations were insensitive to this event. The Tohuvavohu et al. (2019) $u$-band observations could detect a GW190425-like $1.4+2.0 \mathrm{M}_{\odot}$ BNS merger to $D=31 \mathrm{Mpc}$, again indicating that they were not sensitive enough to detect the EM counterpart of GW190425. The Hosseinzadeh et al. (2019) $i$-band observations were sufficiently deep to detect GW190425 to $150 \mathrm{Mpc}$, not quite the median distance, if observed at the time of peak luminosity.

\subsection{Future Searches}

The community's search for an EM counterpart to GW190425 was likely inefficient. The difference in efficiency between the GW190425 search and the extremely successful search for the counterpart of GW170817 is primarily caused by the physical differences of the systems and the likely prompt collapse of the GW190425 system to a black hole. (Differences in success also likely depended on the localization area.) This resulted in an absolute magnitude difference in the $r$ band (the most commonly used filter for searching) of $\Delta M_{r}=3 \mathrm{mag}$. The difference in distance between the two events results in another 1.7-3.8 mag difference. Combined, the EM counterpart of GW190425 was likely 5-7 mag fainter than AT 2017gfo in the $r$ band.

While AT 2017gfo had similar peak luminosities in all optical bands, the EM counterpart to GW190425 was likely much redder having $r-i \approx 1$ mag at peak and $r-i \approx$ $2 \mathrm{mag}$ at $+2 \mathrm{~d}$ after merger $(g-i \approx 1.5$ and $5 \mathrm{mag}$ at comparable times). It is therefore likely more efficient to search in redder bands for similar events, even at the loss of detector sensitivity.

\subsection{Chemical Enrichment}

A BNS or NSBH merger is expected to disperse heavy, $r$-process elements into its environments (Lattimer \& Schramm 1974; Freiburghaus et al. 1999). The rate of enrichment from these events is important for understanding galaxy evolution and if additional sources of $r$-process material are necessary (e.g., Shen et al. 2015; van de Voort et al. 2015, 2019; Naiman et al. 2018).

AT 2017 gfo is often modeled as having two components, each with different lanthanide fractions. For instance, Kilpatrick et al. (2017) modeled AT 2017gfo as having a "red" component with $M_{\mathrm{ej}}=0.035 \mathrm{M}_{\odot}, v=0.15 c$, and $\log X_{\operatorname{lan}}=-2$ and a "blue" component with $M_{\mathrm{ej}}=$ $0.025 \mathrm{M}_{\odot}, v=0.25 c$, and $\log X_{\text {lan }}$ ranging from -4 to -6 . From this, they find a total amount of $r$-process material ejected of $M_{\mathrm{r}-\mathrm{p}} \approx 0.06 \mathrm{M}_{\odot}$. This value is consistent with other estimates (Drout et al. 2017; Kasliwal et al. 2017; Smartt et al. 2017; Tanaka et al. 2017; Tanvir et al. 2017; Villar et al. 2017).

We estimate GW190425 ejected $0.03 \pm 0.01 \mathrm{M}_{\odot}$ of material with $\log X_{\text {lan }} \approx-2$, similar to the "red" component of GW170817/AT 2017gfo, but substantially less than the total mass ejected. This estimate is larger than the stringent minimum mass required to effectively pollute low metallicity, $r$-process enhanced stars (Macias \& Ramirez-Ruiz 2018).

LVC20 determined the individual merger rates for "GW170817-like" and "GW190425-like" events as well as a combined event rate assuming both are drawn from the same population. Following the procedure of Kilpatrick et al. (2017), but using the individual rates and total ejecta masses for each event, we find a total of $5_{-4}^{+11} \times 10^{4} \mathrm{M}_{\odot}$ of $r$-process material produced in a MW-like galaxy over $10^{10} \mathrm{yr}$. This amount is comparable to that estimated from the Solar $r$ process abundance and total mass of stars and gas in the MW (Grevesse et al. 2007; Kafle et al. 2014), indicating that compact-object mergers alone are sufficient for producing the majority of $r$-process material in the Universe.

While GW170817 likely produced both "light" $(A<$ $140)$ and "heavy" $(A>140) r$-process material, GW190425 likely produced primarily heavy $r$-process material. Using a similar argument as above, but using the GW170817-like rate for the blue component of GW170817 and the combined rate for the red component of GW170817 and GW190425, we find compact-object mergers have produced $4_{-2}^{+12} \times 10^{4} \mathrm{M}_{\odot}$ of light and $0.4_{-0.2}^{+1.1} \times 10^{4} \mathrm{M}_{\odot}$ of heavy $r$-process material produced throughout the history of the MW. Roughly $10 \%$ of $r$-process material produced in NS mergers, averaged over all events, is $A>140$ nuclei.

Mergers that eject primarily dynamical material with low $Y_{e}$ are expected to be an excellent site for actinide production, perhaps being a way to produce the abundances seen in "actinide-boost" stars (e.g., Hill et al. 2002). Notably, whatever process enriches actinide-boost stars, it must also produce a similar abundance pattern to the process that enriches normal low-metallicity stars for lighter elements (Roederer et al. 2009). While GW190425-like events may be the source of heavy elements in actinide-boost stars, the 
dynamical ejecta of NSs is expected to overproduce actinides (Holmbeck et al. 2019, although see Wanajo et al. 2014 for a nuanced examination of the $Y_{e}$ distribution in the dynamical ejecta). This may indicate additional components to the GW190425 ejecta or GW190425-like events being rarer in the MW than the measured rate would imply.

\subsection{Expanding the Compact-Object Parameter Space}

The key observation for GW190425 is the high total mass. As we have described in detail, the system could range from an equal-mass BNS system with component masses of $\sim 1.7 \mathrm{M}_{\odot}$ each to a NSBH with $m_{1}=2.4 \mathrm{M}_{\odot}$ and $q=0.5$.

The NSBH scenario requires a BH significantly less massive than the population of known MW BHs (Özel et al. 2010; Farr et al. 2011) and would exist in the "mass gap." However, the mass is so low that it is unlikely to be the result of a previous NS merger. It therefore appears that such a scenario requires producing such a low-mass $\mathrm{BH}$ from core collapse. A population of $\sim 2 \mathrm{M}_{\odot}$ BHs are not currently predicted from most core-collapse simulations (e.g., Ugliano et al. 2012), however stellar evolution modeling indicates a possible pathway (Ertl et al. 2019). Further examination of theoretical models to understand the likelihood of this possibility is required for this scenario to be truly viable.

Additionally, there are only a few simulations of high total mass or extreme mass ratio compact object mergers. Additional simulations of BNS mergers from $1.6+1.6 \mathrm{M}_{\odot}$ to $1.3+2.1 \mathrm{M}_{\odot}$ and NSBH mergers with $1.4+2.0 \mathrm{M}_{\odot}$ to $1.2+2.4 \mathrm{M}_{\odot}$ are necessary to fully understand the properties of the GW190425 ejecta. Future GW events may have slightly higher total mass indicating even higher mass ratios. Extending current merger simulations to higher total masses and more extreme mass ratios will be important for precise modeling of this new population of binary systems.

\section{CONCLUSIONS}

The discovery of GW190425, the second likely BNS merger, was a landmark event, unveiling a new population of binary compact object systems not yet discovered in the MW. We can infer from the exquisite GW data that the total system mass is significantly different from the known population of MW BNSs.

Extending that analysis, we use astrophysical knowledge to further constrain the parameters of GW190425. Depending on the exact assumptions, we can reduce the parameter space to $<30 \%$ that of $\mathrm{LVC} 20$. We detail three relevant scenarios that can then be used in future studies to understand the formation of the progenitor system, focus merger models, and enhance population studies.

If GW190425 was a BNS system where the lighter component has a mass similar to the non-recycled NSs in MW BNS systems, we find $m_{1}=2.03_{-0.14}^{+0.15}, m_{2}=1.35 \pm 0.09$, and $m_{\text {tot }}=3.38_{-0.07}^{+0.08} \mathrm{M}_{\odot}$. Such a system would have a small mass ratio $\left(q=0.67_{-0.08}^{+0.10}\right)$ and a relatively high $\chi_{\mathrm{eff}}$ of $0.05 \pm 0.03$ (all uncertainties being 90th percentile).

However, if we assume that GW190425 had a mass ratio similar to that of MW BNS systems that merge within a Hubble time, we find $m_{1}=1.70_{-0.06}^{+0.17}, m_{2}=1.60_{-0.14}^{+0.05}$, and $m_{\text {tot }}=3.31 \pm 0.05 \mathrm{M}_{\odot}$. This highly constrained system is also expected to have a small $\chi_{\text {eff }}$ of $0.02_{-0.02}^{+0.03}$.

Finally, if GW190425 was a NSBH system, we find $m_{1}=$ $2.19_{-0.17}^{+0.21}, m_{2}=1.26_{-0.08}^{+0.10}$, and $m_{\text {tot }}=3.46_{-0.09}^{+0.13} \mathrm{M}_{\odot}$. This system requires an extreme mass ratio of $q=0.57_{-0.08}^{+0.10}$ and a relatively high $\chi_{\text {eff }}$ of $0.57_{-0.08}^{+0.10}$.

Regardless of the exact scenario, we expect the remnant to either already be a $\mathrm{BH}$ or promptly collapse to a BH. Again, regardless of the system, we expect $\sim 0.03 \mathrm{M}_{\odot}$ of lanthanide-rich ejecta. Using updated event rate estimates and these ejecta characteristics, we find that compact-object mergers produce a significant fraction - and perhaps essentially all $-r$-process material in the Universe.

The expected kilonova associated with GW190425 is expected to have similar NIR properties as AT 2017gfo, but should be significantly fainter in the optical. As a result, we believe essentially no follow-up observation obtained to find the electromagnetic counterpart was constraining.

Optical/infrared searches could choose appropriate filters and adjust exposure times to optimally search for GW170817-like and GW190425-like events if LVC promptly released the chirp mass. This information is available during the initial analysis and would significantly improve chances of detecting counterparts while improving the efficiency of observatories.

\section{ACKNOWLEDGEMENTS}

This work was only possible because of the LVC and its members. Their tireless work operating the GW detectors, analyzing the data, and providing data to the community has sped discovery in the nascent field. We especially thank them for providing the posterior data sets soon after LVC20 was made public.

Much of this manuscript was conceived while R.J.F. attended the Hirschegg 2020 workshop. We thank the organizers and attendees, especially B. Côté, S. De, R. Essick, R. Gamba, G. Raaijmakers, S. Reddy, and L. Roberts for enlightening conversations about GW190425. We also thank P. Brady, D. Lin, P. Macias, B. Margalit, M. Safarzadeh, and S. Woosley for additional help and conversations.

The UCSC team is supported in part by NASA grant NNG17PX03C, NSF grant AST-1815935, the Gordon \& Betty Moore Foundation, the Heising-Simons Foundation, and by fellowships from the Alfred P. Sloan Foundation and the David and Lucile Packard Foundation to R.J.F. E.R.-R. thanks the Heising-Simons Foundation, the Danish National Research Foundation (DNRF132) and NSF (AST-1911206 and AST-1852393) for support. D.A.C. acknowledges support from the National Science Foundation Graduate Research Fellowship under Grant DGE1339067. J.S. is also supported by the A. F. Morrison Fellowship at Lick Observatory.

\section{REFERENCES}

Abbott B. P., et al., 2016, Physical Review Letters, 116, 061102 Abbott B. P., et al., 2017a, Physical Review Letters, 119, 161101 Abbott B. P., et al., 2017b, Nature, 551, 85

Abbott B. P., et al., 2018, Phys. Rev. Lett., 121, 161101 
Abbott B. P., et al., 2019, Physical Review X, 9, 011001

Ai S., Gao H., Zhang B., 2019, arXiv e-prints, p. arXiv:1912.06369

Andreoni I., et al., 2017, Publ. Astron. Soc. Australia, 34, e069

Annala E., Gorda T., Kurkela A., Vuorinen A., 2018, Phys. Rev. Lett., 120, 172703

Antier S., et al., 2019, MNRAS, p. 2740

Antoniadis J., Tauris T. M., Ozel F., Barr E., Champion D. J., Freire P. C. C., 2016, arXiv e-prints, p. arXiv:1605.01665

Ascenzi S., et al., 2019, MNRAS,

Barack L., et al., 2019, Classical and Quantum Gravity, 36, 143001

Bauswein A., Baumgarte T. W., Janka H.-T., 2013, Physical Review Letters, 111, 131101

Bauswein A., Just O., Janka H.-T., Stergioulas N., 2017, ApJ, 850, L34

Blanchard P. K., et al., 2017, ApJ, 848, L22

Coughlin M. W., Dietrich T., Heinzel J., Khetan N., Antier S., Christensen N., Coulter D. A., Foley R. J., 2019, arXiv eprints,

Coulter D. A., et al., 2017, Science, 358, 1556

Cowperthwaite P. S., et al., 2017, ApJ, 848, L17

Cromartie H. T., et al., 2020, Nature Astronomy, 4, 72

De S., Finstad D., Lattimer J. M., Brown D. A., Berger E., Biwer C. M., 2018, Physical Review Letters, 121, 091102

Dietrich T., Samajdar A., Khan S., Johnson-McDaniel N. K., Dudi R., Tichy W., 2019, Phys. Rev. D, 100, 044003

Drout M. R., et al., 2017, Science, 358, 1570

Ertl T., Woosley S. E., Sukhbold T., Janka H. T., 2019, arXiv e-prints, p. arXiv:1910.01641

Evans P. A., et al., 2017, Science, 358, 1565

Farr W. M., Sravan N., Cantrell A., Kreidberg L., Bailyn C. D., Mandel I., Kalogera V., 2011, ApJ, 741, 103

Farrow N., Zhu X.-J., Thrane E., 2019, ApJ, 876, 18

Ferdman R. D., PALFA Collaboration 2018, in Weltevrede P., Perera B. B. P., Preston L. L., Sanidas S., eds, IAU Symposium Vol. 337, Pulsar Astrophysics the Next Fifty Years. pp 146-149, doi:10.1017/S1743921317009139

Ferdman R. D., et al., 2014, MNRAS, 443, 2183

Fernández R., Metzger B. D., 2013, MNRAS, 435, 502

Freiburghaus C., Rosswog S., Thielemann F.-K., 1999, ApJ, 525, L121

Goldstein A., et al., 2017, ApJ, 848, L14

Gompertz B. P., et al., 2018, ApJ, 860, 62

Grevesse N., Asplund M., Sauval A. J., 2007, Space Sci. Rev., 130,105

Guidorzi C., et al., 2017, ApJ, 851, L36

Han M.-Z., Tang S.-P., Hu Y.-M., Li Y.-J., Jiang J.-L., Jin Z.-P., Fan Y.-Z., Wei D.-M., 2020, arXiv e-prints, p. arXiv:2001.07882

Hill V., et al., 2002, A\&A, 387, 560

Holmbeck E. M., Sprouse T. M., Mumpower M. R., Vassh N., Surman R., Beers T. C., Kawano T., 2019, ApJ, 870, 23

Hosseinzadeh G., et al., 2019, ApJ, 880, L4

Kafle P. R., Sharma S., Lewis G. F., Bland-Hawthorn J., 2014, ApJ, 794, 59

Kasen D., Metzger B., Barnes J., Quataert E., Ramirez-Ruiz E., 2017, Nature, 551, 80

Kasliwal M. M., et al., 2017, Science, 358, 1559

Kilpatrick C. D., et al., 2017, Science, 358, 1583

Kitaura F. S., Janka H.-T., Hillebrandt W., 2006, A\&A, 450, 345

Korobkin O., Rosswog S., Arcones A., Winteler C., 2012, MNRAS, 426, 1940

Lattimer J. M., Schramm D. N., 1974, ApJ, 192, L145

Levan A. J., et al., 2017, ApJ, 848, L28

Ligo Scientific Collaboration VIRGO Collaboration 2019a, GRB Coordinates Network, 24168, 1

Ligo Scientific Collaboration VIRGO Collaboration 2019b, GRB Coordinates Network, 24228, 1
Lippuner J., Roberts L. F., 2015, ApJ, 815, 82

Lundquist M. J., et al., 2019, ApJ, 881, L26

Macias P., Ramirez-Ruiz E., 2018, ApJ, 860, 89

Margalit B., Metzger B. D., 2017, ApJ, 850, L19

Martinez J. G., et al., 2015, ApJ, 812, 143

McCully C., et al., 2017, ApJ, 848, L32

Metzger B. D., Fernández R., 2014, MNRAS, 441, 3444

Murguia-Berthier A., Montes G., Ramirez-Ruiz E., De Colle F., Lee W. H., 2014, ApJ, 788, L8

Murguia-Berthier A., et al., 2017, ApJ, 848, L34

Naiman J. P., et al., 2018, MNRAS, 477, 1206

Oppenheimer J. R., Volkoff G. M., 1939, Physical Review, 55, 374

Özel F., Freire P., 2016, ARA\&A, 54, 401

Özel F., Psaltis D., Narayan R., McClintock J. E., 2010, ApJ, 725,1918

Pan Y.-C., et al., 2017, ApJ, 848, L30

Piro A. L., Giacomazzo B., Perna R., 2017, ApJ, 844, L19

Podsiadlowski P., Langer N., Poelarends A. J. T., Rappaport S., Heger A., Pfahl E., 2004, ApJ, 612, 1044

Pozanenko A. S., Minaev P. Y., Grebenev S. A., Chelovekov I. V., 2019, arXiv e-prints, p. arXiv:1912.13112

Radice D., Bernuzzi S., Del Pozzo W., Roberts L. F., Ott C. D., 2017, ApJ, 842, L10

Radice D., Perego A., Zappa F., Bernuzzi S., 2018, ApJ, 852, L29

Ramirez-Ruiz E., Andrews J. J., Schrøder S. L., 2019, ApJ, 883, L6

Roederer I. U., Kratz K.-L., Frebel A., Christlieb N., Pfeiffer B., Cowan J. J., Sneden C., 2009, ApJ, 698, 1963

Romero-Shaw I. M., Farrow N., Stevenson S., Thrane E., Zhu X.-J., 2020, arXiv e-prints, p. arXiv:2001.06492

Rosswog S., 2005, ApJ, 634, 1202

Rosswog S., 2013, Philosophical Transactions of the Royal Society of London Series A, 371, 20120272

Rosswog S., Sollerman J., Feindt U., Goobar A., Korobkin O., Wollaeger R., Fremling C., Kasliwal M. M., 2018, A\&A, 615, A132

Ruiz M., Shapiro S. L., Tsokaros A., 2018, Phys. Rev. D, 97, 021501

Safarzadeh M., Ramirez-Ruiz E., Berger E., 2020, arXiv e-prints, p. arXiv:2001.04502

Shen S., Cooke R. J., Ramirez-Ruiz E., Madau P., Mayer L., Guedes J., 2015, ApJ, 807, 115

Shibata M., Fujibayashi S., Hotokezaka K., Kiuchi K., Kyutoku K., Sekiguchi Y., Tanaka M., 2017, Phys. Rev. D, 96, 123012

Shibata M., Zhou E., Kiuchi K., Fujibayashi S., 2019, Phys. Rev. D, 100, 023015

Siebert M. R., et al., 2017, ApJ, 848, L26

Smartt S. J., et al., 2017, Nature, 551, 75

Song H.-R., Ai S.-K., Wang M.-H., Xing N., Gao H., Zhang B., 2019, ApJ, 881, L40

Sukhbold T., Ertl T., Woosley S. E., Brown J. M., Janka H. T., 2016, ApJ, 821, 38

Suwa Y., Yoshida T., Shibata M., Umeda H., Takahashi K., 2018, MNRAS, 481, 3305

Tanaka M., et al., 2017, PASJ, 69, 102

Tanvir N. R., et al., 2017, ApJ, 848, L27

Tauris T. M., Janka H.-T., 2019, ApJ, 886, L20

The LIGO Scientific Collaboration the Virgo Collaboration 2020, arXiv e-prints, p. arXiv:2001.01761

The LIGO Scientific Collaboration et al., 2018, arXiv e-prints,

Timmes F. X., Woosley S. E., Weaver T. A., 1996, ApJ, 457, 834

Tohuvavohu A., et al., 2019, GRB Coordinates Network, 24353,

Tolman R. C., 1939, Physical Review, 55, 364

Ugliano M., Janka H.-T., Marek A., Arcones A., 2012, ApJ, 757, 69

Utsumi Y., et al., 2017, PASJ, 69, 101

Valenti S., et al., 2017, ApJ, 848, L24 
$10 \quad$ Foley et al.

Villar V. A., et al., 2017, ApJ, 851, L21

Wanajo S., Sekiguchi Y., Nishimura N., Kiuchi K., Kyutoku K., Shibata M., 2014, ApJ, 789, L39

van de Voort F., Quataert E., Hopkins P. F., Kereš D., FaucherGiguère C.-A., 2015, MNRAS, 447, 140

van de Voort F., Pakmor R., Grand R. J. J., Springel V., Gómez

F. A., Marinacci F., 2019, arXiv e-prints, p. arXiv:1907.01557 
Table 1. Source properties for GW190425

\begin{tabular}{|c|c|c|c|c|c|}
\hline Parameter & LVC high-spin prior & BNS & $\mathrm{BNS}+m_{2} \approx M_{\mathrm{Ch}}$ & $\mathrm{BNS}+q \approx 1$ & $\mathrm{NSBH}$ \\
\hline Primary mass $m_{1}\left(\mathrm{M}_{\odot}\right)$ & $2.02_{-0.34}^{+0.58}$ & $1.85_{-0.19}^{+0.27}$ & $2.03_{-0.14}^{+0.15}$ & $1.70_{-0.06}^{+0.17}$ & $2.19_{-0.17}^{+0.21}$ \\
\hline Secondary mass $m_{2}\left(\mathrm{M}_{\odot}\right)$ & $1.35_{-0.27}^{+0.26}$ & $1.47_{-0.18}^{+0.16}$ & $1.35 \pm 0.09$ & $1.60_{-0.14}^{+0.05}$ & $1.26_{-0.08}^{+0.10}$ \\
\hline Chirp mass $\mathcal{M}\left(\mathrm{M}_{\odot}\right)$ & $1.437_{-0.020}^{+0.022}$ & $1.436_{-0.019}^{+0.022}$ & $1.433_{-0.019}^{+0.023}$ & $1.436_{-0.019}^{+0.021}$ & $1.438_{-0.020}^{+0.021}$ \\
\hline Mass ratio $q=m_{2} / m_{1}$ & $0.67_{-0.25}^{+0.29}$ & $0.79_{-0.19}^{+0.18}$ & $0.67_{-0.08}^{+0.10}$ & $0.94_{-0.16}^{+0.05}$ & $0.57_{-0.08}^{+0.10}$ \\
\hline Total mass $m_{\text {tot }}\left(\mathrm{M}_{\odot}\right)$ & $3.39_{-0.11}^{+0.32}$ & $3.33_{-0.06}^{+0.10}$ & $3.38_{-0.07}^{+0.08}$ & $3.31 \pm 0.05$ & $3.46_{-0.09}^{+0.13}$ \\
\hline Effective inspiral spin parameter $\chi_{\text {eff }}$ & $0.06_{-0.05}^{+0.11}$ & $0.03_{-0.03}^{+0.04}$ & $0.05 \pm 0.03$ & $0.02_{-0.02}^{+0.03}$ & $0.07_{-0.03}^{+0.04}$ \\
\hline Luminosity distance $D_{L}(\mathrm{Mpc})$ & $159_{-71}^{+69}$ & $162_{-73}^{+67}$ & $171_{-76}^{+66}$ & $161_{-70}^{+66}$ & $154_{-69}^{+70}$ \\
\hline
\end{tabular}

